\title{
Phoniatry
}

\section{Stabilometric findings in patients affected by organic dysphonia before and after phonomicrosurgery}

\author{
Analisi posturografica in pazienti con disfonia organica prima e dopo \\ fonomicrochirurgia
}

\author{
A. NACCl ${ }^{1}$, S.O. ROMEO ${ }^{1}$, S. BERRETTINI ${ }^{12}$, J. MATTEUCCl ${ }^{1}$, M.D. CAVALIERE ${ }^{1}$, V. MANCINI ${ }^{1}$, E. PANICUCCl $^{3}$, \\ F. URSINO ${ }^{4}$, B. FATTORI \\ ${ }^{1}$ ENT Audiology Phoniatrics Unit, Department of Neurosciences, University of Pisa, Italy; ${ }^{2}$ Division of ENT Diseases, \\ Karolinska Institutet, Stockholm, Sweden; ${ }^{3}$ Department of Surgical, Medical and Molecular Pathology, University of \\ Pisa, Italy; ${ }^{4}$ National Institute for Research in Phoniatrics, University of Pisa, Italy
}

\begin{abstract}
SUMMARY
The purpose of this study is to understand if there is any alteration in the posture of patients affected by organic dysphonia and describe possible postural modifications after phonomicrosurgery on the vocal folds. Forty subjects ( 22 males, 18 females; mean age $32.6 \pm 7.5$ years) suffering from organic dysphonia (15 cases of polyps, 11 submucosal retention cysts, 10 bilateral fibrous vocal fold nodules and 4 bilateral Reinke's oedema) were examined by open-eye and closed-eye posturography while breathing spontaneously before surgery, 24 hours after surgery and after 6 months. The variables taken into account were: the coordinates of the centre of pressure on both frontal and sagittal planes, length and surface of the track, mean velocity of the oscillations and relative standard deviations, spectral analysis of oscillation frequency, statokinesigram and stabilogram values. No characteristic pathological pattern was seen in basal stabilometry in any of the subgroups (polyps, cysts, Reinke's oedema). Only the subgroup of patients with fibrous vocal fold nodules (8/10; 80\%) showed a slight forward shift from the centre of gravity when analysed in both open-eye and closed-eye posturography. A comparison performed within the same subgroup using open-eye and closed-eye posturography before and after surgery revealed no significant difference in any of the parameters being studied. The use of static stabilometry in this study demonstrates the absence of characteristic postural alterations in patients affected by organic dysphonia and also excludes that simple removal of the vocal fold lesion can change posture.
\end{abstract}

KEY WORDS: Posture $\bullet$ Static stabilometry $\bullet$ Proprioception $\bullet$ Phonomicrosurgery $\bullet$ Organic voice disorders

\section{RIASSUNTO}

Lo scopo di questo studio è stato quello di dimostrare se pazienti affetti da disfonia organica presentino alterazioni posturali caratteristiche e se l'intervento di fonomicrochirurgia delle corde vocali determini modificazioni dell'assetto posturale. Sono stati esaminati 40 soggetti (22 maschi, 18 femmine; età media 32,6 \pm 7,5 anni) affetti da disfonia organica (15 polipi cordali, 11 cisti da ritenzione sottomucosa, 10 noduli vocali fibrosi bilaterali e 4 edema di Reinke). L'assetto posturale è stato studiato mediante stabilometria statica in respirazione spontanea, ad occhi aperti e ad occhi chiusi, in tre diverse fasi: prima dell'intervento chirurgico, 24 ore dopo la chirurgia e 6 mesi dopo l'intervento. Le variabili prese in considerazione sono state: le coordinate del centro di pressione sul piano frontale e sagittale, la lunghezza e la superficie della traccia, la velocità media delle oscillazioni e le relative deviazioni standard, l'analisi spettrale della frequenza di oscillazione e $i$ valori di statokinesigramma e stabilogramma. Nessun pattern patologico caratteristico veniva rilevato alla stabilometria in condizioni basali nei diversi sottogruppi di pazienti (polipi, cisti, edema di Reinke). Solo il sottogruppo di pazienti con noduli vocali bilaterali fibrosi (8/10; 80\%) mostrava un lieve spostamento in avanti del centro di gravità sia ad occhi aperti che ad occhi chiusi. In tutti $i$ sottogruppi non si verificavano modificazioni significative delle variabili stabilometriche ad occhi aperti e ad occhi chiusi, prima e dopo l'intervento chirurgico. L'uso della stabilometria statica, in questo studio, dimostra l'assenza di alterazioni posturali caratteristiche nei casi di disfonia organica ed esclude che la semplice rimozione della lesione cordale, unilaterale o bilaterale, possa determinare variazioni posturali.

PAROLE CHIAVE: Postura $\bullet$ Stabilometria statica $\bullet$ Propriocezione $\bullet$ Fonomicrochirurgia $\bullet$ Disfonia organica

Acta Otorhinolaryngol Ital 2017;37:286-294

\section{Introduction}

There is general agreement in the literature that posture control and voice are related ${ }^{1-3}$; in fact, it has been demonstrated that correct posture is fundamental for quality function of the voice ${ }^{4-12}$. Voice disorders are usually associated with alterations in extra-laryngeal muscle activity, whereas changes in posture can sometimes have a significant role in the genesis and/or maintenance of these voice disorders, in a sort of vicious circle where a change in 
extra-laryngeal tension and an inadequate activity of the larynx itself contributes to and influences each other ${ }^{13}$. In recent years, the use of stabilometry for studying posture has permitted overcoming the many limits given by clinical tests alone ${ }^{311-14}$. Computerised posturographic studies performed with a stabilometric platform supply extremely sensitive numeric parameters in subjects with purely dysfunctional dysphonia or dysphonia associated with secondary organic disorders, and even in subjects with no primary balance problems. Moreover, using static stabilometry in open-eye, closed-eye and in closed-eye with head backwards, the importance of the individual sensorial inputs (visual, vestibular and cervical proprioceptive) can be assessed, and when the test is performed during natural breathing (not forced) it is possible to study the prevalently reflex component of posture (spinal vestibular reflex: SVR) ${ }^{13}$. In fact, the systems controlling posture, balance and organisation of body movement rely principally on the integration of specific sensorial patterns for their function, followed by the elaboration of specific motor responses that are mainly reflex ones (the most important of which is undoubtedly the SVR).

Our recent studies on subjects with pure dysfunctional dysphonia show how the greater proprioception achieved through speech rehabilitation brings an improvement in the majority of the postural parameters evaluated with static stabilometry ${ }^{13}$. Our studies confirmed what was previously described in the relevant literature. ${ }^{3}$ One of these studies evaluated postural variations in a group of subjects with dysfunctional dysphonia, and then compared these variations with the modifications seen in the stabilometric parameters after speech therapy ${ }^{13}$. The results obtained were very encouraging and call attention not only to the importance of stabilometric analysis in subjects with voice disorders associated with postural changes, but also to the fundamental role rehabilitation plays when resolving dysphonia and at the same time improving postural performance. With this in mind, investigation of the postural characteristics of patients with voice disorders who have organic lesions in the vocal folds appears interesting. We therefore submitted subjects with organic dysphonia due to acquired, benign vocal fold lesions to posturographic analysis, performing static stabilometry before and after phonomicrosurgery. Our aim was to understand if there were any postural characteristics particular to the individual organic disorders and to assess if removal of the lesion with phonomicrosurgery is, on its own, sufficient to modify the postural performance of subjects.

\section{Materials and methods}

In this study, we assessed 40 patients (22 males and 18 females; mean age $32.6 \pm 7.5$ years) with organic dysphonia: 15 had a vocal fold polyp, 11 a submucosal retention cyst, 10 bilateral fibrous vocal fold nodules and 4 cases of bilateral Reinke's oedema. The clinical characteristics of patients are summarised in Table I. All subjects were submitted to a posturographic test using a static stabilometric platform (S.Ve.P. AMPLIFON-Amplaid, Milan, Italy), inviting patients to stand upright during the test and breathe normally. The test was carried out with the patients' eyes both open (OE) and closed (CE) to eliminate visual afferents and enhance proprioceptive faculties. The posturographic test was carried out in basal conditions and then repeated 24 hours after phonosurgical removal of the vocal fold lesion. Moreover, the posturographic test was repeated at 6 months after surgery under the same conditions as the baseline and 24 hour control. The following parameters were considered: pressure centre coordinates on the frontal (X; right-left) (minimum $\mathrm{X}$, maximum $\mathrm{X}$, mean X) and sagittal (Y; forward/backward) (minimum Y, maximum $\mathrm{Y}$, mean $\mathrm{Y}$ ) areas; total length of the recording in $\mathrm{mm}(\mathrm{L})$; surface of the helix containing $90 \%$ of the points sampled (S); mean velocity (VEL) and relative SD; Fourier fast transform (FFT) for oscillations on the $\mathrm{X}$ and $\mathrm{Y}$ axes separately; statokinesigram describing the area within which the centre of gravity projects with respect to the floor, and stabilogram which reveals the time taken for the pressure centre to shift on both the $\mathrm{X}$ and $\mathrm{Y}$ axes. Each patient underwent accurate phoniatric and otorhinolaryngoiatric anamnesis, general ENT examination,

Table I. Clinical characteristics of the study group.

\begin{tabular}{|c|c|c|c|c|}
\hline \multirow[t]{2}{*}{ Study Group } & Patients & $\operatorname{Sex}(M / F)$ & Age (yrs) & $\mathrm{LPR}^{\star}$ \\
\hline & $\mathrm{n}=40$ & $22 \mathrm{M} / 18 \mathrm{~F}$ & $32.6 \pm 7.5$ & NO \\
\hline Subgroups & Patients & Sex (M/F) & Age (yrs) & $\mathrm{LPR}^{*}$ \\
\hline Polyps & $\begin{array}{c}n=15 \\
15 / 40(37.5 \%)\end{array}$ & $10 \mathrm{M} / 5 \mathrm{~F}$ & $29.9 \pm 6.4$ & NO \\
\hline Acquired cysts & $\begin{array}{c}n=11 \\
11 / 40(27.5 \%)\end{array}$ & $7 \mathrm{M} / 4 \mathrm{~F}$ & $32.1 \pm 7.3$ & NO \\
\hline Bilateral nodules & $\begin{array}{c}n=10 \\
10 / 40(25 \%)\end{array}$ & $2 \mathrm{M} / 8 \mathrm{~F}$ & $33.3 \pm 6.6$ & NO \\
\hline Reinke's oedema & $\begin{array}{c}n=4 \\
4 / 40(10 \%)\end{array}$ & $3 \mathrm{M} / 1 \mathrm{~F}$ & $42.8 \pm 7.4$ & NO \\
\hline
\end{tabular}


flexible fiberoptic rhino-pharyngo-laryngoscopy, videolaryngostroboscopy, electro-acoustic voice tests including spectrography and analysis of the vocal signal with the multidimensional voice program (MDVP). Vocal signal was acquired by asking the patient to pronounce a sustained [a] vowel. All patients had been suffering from chronic dysphonia for at least six months and diagnosis of organic lesion of the vocal fold was made for at least three months in all study subjects.

All patients remarked that their voice disorder limited their social or working life. The subjective degree of severity of the condition was evaluated using the Voice Handicap Index (VHI) questionnaire, which each patient filled in before surgical treatment ${ }^{15}$. The perceptive evaluation of voice was scored against the GRBAS Scale ${ }^{16}$. The acoustic analysis, perceptual analysis and patient's self-assessment were performed before and 6 months after surgery.

The tests carried out with flexible fiberoptic endoscopy and rigid videolaryngostroboscopy $\left(70^{\circ}\right)$ revealed organic damage in the glottic plane of all the subjects in the study. None of the patients showed signs of posterior inflammation due to laryngo-pharyngeal reflux, whereas they all showed some degree of tentative compensation by the supraglottic structures to aid closure of the glottis during phonation, since closure was faulty because of the organic lesion on the vocal fold. In the 5 cases with submucosal cyst, stroboscopic light revealed an interruption of mucosal wave on the site of the lesion.

After accurate anamnesis and orthopaedic examination, all patients with orthopaedic disorders or known postural defects (varus/valgus foot, scoliosis, previous orthopaedic surgery of any kind, etc.) were excluded from the study. Additionally, those who showed balance disorders during anamnesis and/or were classified positive upon otoneurological examination were excluded from the study. To evaluate the possible presence of oto-neurological diseases, the following tests were performed: accurate oto-neurological assessment, liminal tonal audiometry, investigation of spontaneous and provoked nystagmus by videonystagmography and vestibular caloric balance. Other exclusion criteria were systemic disease, alcohol abuse, psychiatric disorder and vision less than 10/10.

Seven subjects were excluded from the study: 3 cases were affected by oto-neurological diseases ( 1 case of Menière's disease, 1 case of recurrent paroxysmal positional vertigo and 1 case of recent vestibular neuronitis), 2 cases were affected by scoliosis, 1 case was affected by whiplash injury with functional limitation of the cervical spine, while 1 case was affected by diabetes mellitus type I. Therefore, considering the inclusion and exclusion criteria, 40 subjects were included in the study group.

None of the patients had undergone previous speech rehabilitation/therapy. Even the 10 patients with fibrous nodules had never been submitted to rehabilitation treatment since, at diagnosis, patients chose surgical treatment after all therapeutic options had been explained to them.
After surgical treatment, the study group was not submitted to speech therapy either because it was not advisable or because the patients refused it.

\section{Surgical treatment}

The phonomicrosurgery procedure was performed under general anaesthesia using specific instruments for endolaryngeal phonosurgery and according to the methods described in the national and international literature ${ }^{17} 18$.

\section{Statistical analysis}

All numeric parameters are expressed in medians. A comparison was performed between several parameters at different times, before and after surgical treatment. The comparison (before open-eye $v s$ after open-eye mode; before closed-eye $v s$ after closed-eye mode; after $24 \mathrm{~h} v s$ after 6 months surgery) was carried out considering the median of the different parameters with a Wilcoxon nonparametric test and considering the same parameters for the percentage of pathological patients using a chi ${ }^{2}$ Test. The parameters resulting above normal range with this instrument were considered pathological. All tests were considered significant with $\mathrm{p}<0.05$. StatView 5 release 5.0.1 was used to process data.

\section{Results}

Control laryngostroboscopy carried out at one month and 6 months after phonomicrosurgery demonstrated the absence of organic lesions in all the subjects.

The results of acoustic analysis (MDVP), perceptual analysis (GRBAS) and VHI performed before and 6 months after surgical treatment are summarised in Tables II, III and IV.

\section{Stabilometric study}

\section{Basal conditions (Pre-OE and Pre-CE)}

In basal conditions (before phonomicrosurgery), no characteristic pathological stabilometric pattern was seen within the individual groups (polyps, cysts and Reinke's oedema). An anterior shift from the centre of gravity was seen in 8 of 10 patients with fibrous vocal nodules $(80 \%)$ in both open-eye and closed-eye tests. Such an anterior shift from the centre of gravity was evident at analysis of the posturographic trace and, in particular, of the statokinesigram.

Pre-OE vs Post-OE1 and Pre-CE vs Post-CE1 (Follow-up at $24 \mathrm{~h}$ ) and Pre-OE vs Post-OE2 and Pre-CE vs PostCE2 (follow-up at 6 months)

A comparison performed within the individual groups (polyps, nodules, cysts and Reinke's oedema) before and after surgery (after 24 hours and after 6 months) in open-eye (Pre-OE vs Post-OE1; Pre-OE vs Post-OE2) and 
Table II. Results of the acoustic analysis (MDVP) for Jitter \%, Shimmer \%, NHR and SPI parameters, before and 6 months after phonomicrosurgery.

\begin{tabular}{|c|c|c|c|}
\hline \multicolumn{4}{|c|}{ POLYPS } \\
\hline & Pre & Post & $p$ \\
\hline Jitter \% & $2.23 \pm 0.32$ & $0.31 \pm 0.36$ & $p<0.01$ \\
\hline Shimmer \% & $3.34 \pm 0.82$ & $1.92 \pm 0.71$ & \\
\hline NHR & $0.14 \pm 0.12$ & $0.09 \pm 0.06$ & \\
\hline SPI & $26.43 \pm 4.24$ & $8.11 \pm 4.13$ & \\
\hline \multicolumn{4}{|c|}{ NODULES } \\
\hline & Pre & Post & $p$ \\
\hline Jitter \% & $1.73 \pm 0.21$ & $0.91 \pm 0.22$ & $p<0.01$ \\
\hline Shimmer \% & $4.15 \pm 0.84$ & $2.13 \pm 0.41$ & \\
\hline NHR & $0.12 \pm 0.02$ & $0.06 \pm 0.02$ & \\
\hline SPI & $16.36 \pm 3.83$ & $4.92 \pm 1.63$ & \\
\hline \multicolumn{4}{|c|}{ CYSTS } \\
\hline & Pre & Post & $p$ \\
\hline Jitter \% & $1.97 \pm 0.44$ & $0.52 \pm 0.31$ & $p<0.01$ \\
\hline Shimmer \% & $5.26 \pm 0.85$ & $0.33 \pm 0.01$ & \\
\hline NHR & $0.18 \pm 0.31$ & $0.08 \pm 0.06$ & \\
\hline SPI & $16.94 \pm 3.93$ & $11.12 \pm 1.63$ & \\
\hline \multicolumn{4}{|c|}{ REINKE'S OEDEMA } \\
\hline & Pre & Post & $p$ \\
\hline Jitter \% & $4.55 \pm 1.25$ & $1.23 \pm 0.61$ & $p<0.01$ \\
\hline Shimmer \% & $11.46 \pm 3.23$ & $3.82 \pm 1.31$ & \\
\hline $\mathrm{NHR}$ & $0.25 \pm 0.14$ & $0.12 \pm 0.04$ & \\
\hline SPI & $12.86 \pm 3.67$ & $6.25 \pm 1.24$ & \\
\hline
\end{tabular}

closed-eye manner (Pre-CE vs Post-CE1; Pre-CE vs PostCE2) showed that there were no statistically significant differences in any of the parameters studied. Even the stabilometric tests performed after surgery in the 10 subjects with vocal nodules detected no shifts from the centre of gravity and showed no substantial modifications compared to basal conditions in the parameters studied.

\section{Post-OE1 (24h) vs Post-OE2 (6 months) and Post-CE1} (24h) vs Post-CE2 (6 months)

A comparison performed within the individual groups after surgery (24h) vs follow-up (6 months) in open-eye (Post-OE1 vs Post-OE2) and closed-eye manner (PostCE1 vs Post-CE2) showed that there were no statistically significant differences in any of the parameters studied.
Table IV. Results of the perceptual analysis (GRBAS) before and 6 months after phonomicrosurgery.

\begin{tabular}{|c|c|c|c|}
\hline \multicolumn{4}{|c|}{ POLYPS } \\
\hline & Pre & Post & $p$ \\
\hline G & $1.7 \pm 0.6$ & $0.2 \pm 0.3$ & $p<0.0001$ \\
\hline $\mathrm{R}$ & $1.2 \pm 0.5$ & $0.1 \pm 0.2$ & \\
\hline B & $1.4 \pm 0.6$ & $0.1 \pm 0.2$ & \\
\hline A & $0.9 \pm 0.3$ & $0.1 \pm 0.2$ & \\
\hline S & $0.7 \pm 0.6$ & $0.2 \pm 0.3$ & $p=0.04$ \\
\hline \multicolumn{4}{|c|}{ NODULES } \\
\hline & Pre & Post & $p$ \\
\hline G & $1.4 \pm 0.4$ & $0.2 \pm 0.3$ & $p<0.001$ \\
\hline $\mathrm{R}$ & $1.2 \pm 0.3$ & $0.1 \pm 0.1$ & \\
\hline B & $1.7 \pm 0.6$ & $0.2 \pm 0.2$ & \\
\hline A & $1.1 \pm 0.8$ & $0.2 \pm 0.4$ & \\
\hline S & $1.4 \pm 0.6$ & $0.2 \pm 0.2$ & \\
\hline \multicolumn{4}{|c|}{ CYSTS } \\
\hline & Pre & Post & $p$ \\
\hline G & $1.9 \pm 0.8$ & $0.3 \pm 0.4$ & $p<0.001$ \\
\hline $\mathrm{R}$ & $1.5 \pm 0.7$ & $0.3 \pm 0.5$ & \\
\hline B & $1.6 \pm 0.6$ & $0.2 \pm 0.2$ & \\
\hline A & $1.2 \pm 0.4$ & $0.1 \pm 0.2$ & \\
\hline S & $1.1 \pm 0.8$ & $0.2 \pm 0.2$ & \\
\hline \multicolumn{4}{|c|}{ REINKE'S OEDEMA } \\
\hline & Pre & Post & $p$ \\
\hline G & $1.9 \pm 0.7$ & $0.4 \pm 0.1$ & $p<0.005$ \\
\hline $\mathrm{R}$ & $2.0 \pm 0.5$ & $0.5 \pm 0.5$ & \\
\hline B & $0.6 \pm 0.1$ & $0.2 \pm 0.2$ & NS \\
\hline A & $1.2 \pm 0.5$ & $0.3 \pm 0.1$ & $p<0.005$ \\
\hline S & $1.5 \pm 0.6$ & $0.3 \pm 0.2$ & \\
\hline
\end{tabular}

The results are summarised for each subgroup in Tables V and VI.

\section{Discussion}

It is now acknowledged that pure dysfunctional dysphonia or the types associated with secondary larynx disorders (with vocal fold lesions) are related to modified respiratory dynamics and particular postural characteristics ${ }^{19}$. Thanks to computerised posturographic studies, it is now possible to accurately evaluate the numerical parameters and graphics that reflect even the slightest modification

Table III. Results of patient self-assessment (VHI), before and 6 months after phonomicrosurgery.

\begin{tabular}{lccc} 
& Pre & Post & $p$ \\
Polyps & $37.2 \pm 16.6(18-73)$ & $9.3 \pm 3.2(4-14)$ & $p<0.0001$ \\
Nodules & $32.1 \pm 12.5(15-56)$ & $11.8 \pm 5.3(4-19)$ & $p<0.001$ \\
Cysts & $41.5 \pm 15.9(22-71)$ & $10.9 \pm 4.9(3-20)$ & $p<0.0001$ \\
Reinke's oedema & $33.3 \pm 6.7(27-40)$ & $16.8 \pm 2.8(14-20)$ & $p=0.004$ \\
Total $(\mathrm{N}=40)$ & $36.7 \pm 14.7(15-73)$ & $11.1 \pm 4.5(3-20)$ & $p<0.0001$ \\
\hline
\end{tabular}


Table V. Pre-OE, Post-OE1 (after 24h), Post-0E2 (after 6 months): values of each parameter in the open-eye mode study, before and after phonomicrosurgery (median). Pre-OE N (\%), Post-0E1 (after 24h) and Post-0E2 (after 6 months) N (\%): number of pathological patients and the relative percentage for each parameter. p1: statistical significance of the pre- vs post-therapy comparison referring to the median (Wilcoxon). p2: statistical significance of the pre- vs post-therapy comparison referring to the number of pathological patients (Chi²).

\begin{tabular}{|c|c|c|c|c|c|c|c|c|}
\hline \multicolumn{9}{|c|}{ POLYPS $(N=15)$} \\
\hline & Pre-0E & Post-0E1 & Post-0E2 & $\mathrm{p} 1$ & Pre-OE N $(\%)$ & Post-0E1 Nº (\%) & Post-0E2 $N^{\circ}(\%)$ & p2 \\
\hline$X \min$ & -9.89 & -8.56 & -10.1 & NS & $1(6 \%)$ & $2(12 \%)$ & $1(6 \%)$ & NS \\
\hline$X \max$ & 5.21 & 3.49 & 4.24 & NS & $2(12 \%)$ & $1(6 \%)$ & $1(6 \%)$ & NS \\
\hline Xmed & -2.89 & -3.28 & -2.48 & NS & $1(6 \%)$ & $1(6 \%)$ & $1(6 \%)$ & NS \\
\hline $\mathrm{SD}(X)$ & 0.23 & 0.22 & 0.24 & NS & - & - & - & - \\
\hline Ymin & -34.62 & -30.57 & -33.61 & NS & $1(6 \%)$ & 0 & 0 & NS \\
\hline Ymax & -29.67 & -27.93 & -30.35 & NS & 0 & 0 & 0 & NS \\
\hline Ymed & -32.59 & -34.51 & -32.81 & NS & 0 & 0 & 0 & - \\
\hline$S D(Y)$ & 0.29 & 0.27 & 0.32 & NS & - & - & - & NS \\
\hline $\mathrm{L}$ & 194.45 & 201.56 & 203.39 & NS & $1(6 \%)$ & $1(6 \%)$ & 0 & NS \\
\hline S & 141.78 & 135.69 & 138.93 & NS & $2(12 \%)$ & $2(12 \%)$ & $2(12 \%)$ & NS \\
\hline FFTx & 0.04 & 0.04 & 0.04 & NS & - & - & - & - \\
\hline FFTy & 0.08 & 0.04 & 0.04 & NS & - & - & - & - \\
\hline VEL & 12.44 & 11.35 & 13.82 & NS & - & - & - & - \\
\hline SD(VEL) & 4.43 & 4.09 & 4.93 & NS & $3(18 \%)$ & $2(12 \%)$ & $2(12 \%)$ & NS \\
\hline \multicolumn{9}{|c|}{ NODULES $(\mathrm{N}=10)$} \\
\hline & Pre-0E & Post-0E1 & Post-0E2 & $\mathrm{p} 1$ & Pre-0E Nº(\%) & Post-0E1 N(\%) & Post-0E2 N(\%) & p2 \\
\hline Xmin & -9.79 & -9.54 & -10.56 & NS & $2(20 \%)$ & $2(20 \%)$ & $1(10 \%)$ & NS \\
\hline$X \max$ & 4,11 & 3.82 & 5.34 & NS & $1(10 \%)$ & $1(10 \%)$ & $1(10 \%)$ & NS \\
\hline Xmed & -1.39 & -1.03 & -2.62 & NS & $1(10 \%)$ & $1(10 \%)$ & $1(10 \%)$ & NS \\
\hline $\mathrm{SD}(X)$ & 0.26 & 0.24 & 0.22 & NS & - & - & - & - \\
\hline Ymin & -57.52 & -55.31 & -52.83 & NS & $1(10 \%)$ & $1(10 \%)$ & $1(10 \%)$ & NS \\
\hline Ymax & -38.02 & -34.80 & -36.81 & NS & $2(20 \%)$ & $2(20 \%)$ & $2(20 \%)$ & NS \\
\hline Ymed & -47.34 & -45.59 & -44.21 & NS & $1(10 \%)$ & $1(10 \%)$ & $2(20 \%)$ & - \\
\hline$S D(Y)$ & 0.32 & 0.30 & 0.30 & NS & - & - & - & NS \\
\hline $\mathrm{L}$ & 252.36 & 248.81 & 261.78 & NS & $1(10 \%)$ & $1(10 \%)$ & $1(10 \%)$ & NS \\
\hline S & 150.12 & 148.9 & 154.87 & NS & $3(30 \%)$ & $3(30 \%)$ & $3(30 \%)$ & NS \\
\hline FFTx & 0.08 & 0.08 & 0.08 & NS & - & - & - & - \\
\hline FFTy & 0.08 & 0.08 & 0.08 & NS & - & - & - & - \\
\hline VEL & 11.65 & 11.92 & 13.03 & NS & - & - & - & - \\
\hline SD(VEL) & 11.33 & 10.52 & 11.04 & NS & $4(40 \%)$ & $3(30 \%)$ & $3(30 \%)$ & NS \\
\hline \multicolumn{9}{|c|}{ CYSTS (N = 11) } \\
\hline & Pre-0E & Post-0E1 & Post-0E2 & $\mathrm{p} 1$ & Pre-0E Nº(\%) & Post-0E1 No(\%) & Post-0E2 Nº\%) & p2 \\
\hline Xmin & 2.44 & 3.69 & 4.35 & NS & $1(9 \%)$ & $1(9 \%)$ & 0 & NS \\
\hline Xmax & 12.69 & 14.82 & 16.92 & NS & $2(18 \%)$ & $1(9 \%)$ & $1(9 \%)$ & NS \\
\hline Xmed & 8.51 & 6.91 & 8.34 & NS & $1(9 \%)$ & $1(9 \%)$ & $1(9 \%)$ & NS \\
\hline $\mathrm{SD}(X)$ & 0.30 & 0.29 & 0.24 & NS & - & - & - & - \\
\hline Ymin & -49.50 & -44.30 & -51.91 & NS & 0 & 0 & 0 & NS \\
\hline Ymax & -31.39 & -38.31 & -43.72 & NS & $1(9 \%)$ & $2(18 \%)$ & $1(9 \%)$ & NS \\
\hline Ymed & -40.49 & -41.08 & -44.45 & NS & 0 & 0 & 0 & - \\
\hline$S D(Y)$ & 0.33 & 0.31 & 0.30 & NS & - & - & - & NS \\
\hline $\mathrm{L}$ & 229.31 & 212.83 & 259.93 & NS & $1(9 \%)$ & $1(9 \%)$ & $1(9 \%)$ & NS \\
\hline S & 132.28 & 149.72 & 151.78 & NS & $2(18 \%)$ & $1(9 \%)$ & $2(18 \%)$ & NS \\
\hline FFTx & 0.04 & 0.06 & 0.08 & NS & - & - & - & - \\
\hline FFTy & 0.04 & 0.04 & 0.04 & NS & - & - & - & - \\
\hline VEL & 11.56 & 12.83 & 13.35 & NS & - & - & - & - \\
\hline SD(VEL) & 6.89 & 7.31 & 5.21 & NS & $2(18 \%)$ & $2(18 \%)$ & $1(9 \%)$ & NS \\
\hline \multicolumn{9}{|c|}{ Tab. V continues } \\
\hline 290 & & & & & & & & \\
\hline
\end{tabular}


Tab. V follows

\begin{tabular}{|c|c|c|c|c|c|c|c|c|}
\hline \multicolumn{9}{|c|}{ REINKE'S OEDEMA ( $\mathrm{N}=4)$} \\
\hline & Pre-0E1 & Post-0E1 & Post-0E2 & $\mathrm{p} 1$ & Pre-0E $N^{\circ}(\%)$ & Post-0E1 N (\%) & Post-0E2 $N^{\circ}(\%)$ & p2 \\
\hline$X \min$ & -6.32 & -7.02 & -9.23 & NS & 0 & 0 & 0 & NS \\
\hline Xmax & 3.68 & 2.07 & 4.45 & NS & 0 & 0 & 0 & NS \\
\hline Xmed & -1.02 & -2.82 & -3.69 & NS & $1(25 \%)$ & $1(25 \%)$ & 0 & NS \\
\hline $\operatorname{SD}(X)$ & 0.20 & 0.22 & 0.20 & NS & - & - & - & - \\
\hline Ymin & -51.2 & -50.79 & -59.38 & NS & $1(25 \%)$ & $1(25 \%)$ & $1(25 \%)$ & NS \\
\hline Ymax & -31.30 & -26.89 & -26.51 & NS & 0 & 0 & 0 & NS \\
\hline Ymed & -45.19 & -41.79 & -49.42 & NS & 0 & 0 & 0 & - \\
\hline $\mathrm{SD}(\mathrm{Y})$ & 0.39 & 0.37 & 0.35 & NS & - & - & - & NS \\
\hline L & 239.06 & 230.92 & 221.38 & NS & $1(25 \%)$ & $1(25 \%)$ & - & NS \\
\hline$S$ & 108.72 & 121.28 & 118.92 & NS & 0 & 0 & 0 & NS \\
\hline FFTx & 0.04 & 0.04 & 0.04 & NS & - & - & - & - \\
\hline FFTy & 0.04 & 0.04 & 0.04 & NS & - & - & - & - \\
\hline VEL & 11.44 & 10.72 & 15.59 & NS & - & - & - & - \\
\hline SD(VEL) & 7.37 & 6.81 & 8.12 & NS & $2(50 \%)$ & $2(50 \%)$ & $1(25 \%)$ & NS \\
\hline
\end{tabular}

Table VI. Pre- and Post- therapy values considering the above parameters in the closed-eye mode (CE).

\begin{tabular}{|c|c|c|c|c|c|c|c|c|}
\hline \multicolumn{9}{|c|}{ POLYPS (N=15) } \\
\hline & Pre-CE & Post-CE1 & Post-CE2 & p1 & Pre-CE $N^{\circ}(\%)$ & Post-CE1 $N^{\circ}(\%)$ & Post-CE2 $N^{\circ}(\%)$ & p2 \\
\hline Xmin & -14.25 & -12.97 & -10.12 & NS & $1(6 \%)$ & $1(6 \%)$ & 0 & NS \\
\hline$X \max$ & 7.29 & 6.92 & 12.56 & NS & $2(12 \%)$ & $2(12 \%)$ & $1(6 \%)$ & NS \\
\hline Xmed & -4.38 & -3.83 & -1.98 & NS & $1(6 \%)$ & $1(6 \%)$ & $2(12 \%)$ & NS \\
\hline $\mathrm{SD}(X)$ & 0.30 & 0.28 & 0.35 & NS & - & - & - & - \\
\hline Ymin & -47.49 & -45.41 & -54.36 & NS & $1(6 \%)$ & $1(6 \%)$ & $1(6 \%)$ & NS \\
\hline Ymax & -41.31 & -40.29 & -48.05 & NS & $1(6 \%)$ & $1(6 \%)$ & $1(6 \%)$ & NS \\
\hline Ymed & -45.21 & -40.49 & -52.79 & NS & 0 & 0 & 0 & - \\
\hline$S D(Y)$ & 0.30 & 0.29 & 0.30 & NS & - & - & - & NS \\
\hline L & 403.38 & 383.549 & 409.93 & NS & $2(12 \%)$ & $2(12 \%)$ & $2(12 \%)$ & NS \\
\hline S & 326.45 & 319.38 & 312.57 & NS & $2(12 \%)$ & $1(6 \%)$ & $1(6 \%)$ & NS \\
\hline FFTx & 0.04 & 0.04 & 0.04 & NS & - & - & - & - \\
\hline FFTy & 0.08 & 0.08 & 0.06 & NS & - & - & - & - \\
\hline VEL & 15.36 & 14.85 & 18.45 & NS & - & - & - & - \\
\hline SD(VEL) & 9.45 & 8.87 & 10.45 & NS & $3(18 \%)$ & $3(18 \%)$ & $2(12 \%)$ & NS \\
\hline \multicolumn{9}{|c|}{ NODULES $(\mathrm{N}=10)$} \\
\hline & Pre-CE & Post-CE1 & Post-CE2 & p1 & Pre-CE No(\%) & Post-CE1 No(\%) & Post-CE2 $N^{\circ}(\%)$ & p2 \\
\hline Xmin & -16.78 & -15.38 & -17.45 & NS & $1(10 \%)$ & $2(20 \%)$ & $2(20 \%)$ & NS \\
\hline$X \max$ & 8.31 & 7.46 & 9.69 & NS & $2(20 \%)$ & $1(10 \%)$ & $2(20 \%)$ & NS \\
\hline Xmed & -5.38 & -4.13 & -6.38 & NS & $2(20 \%)$ & $2(20 \%)$ & $2(20 \%)$ & NS \\
\hline $\mathrm{SD}(X)$ & 0.40 & 0.38 & 0.40 & NS & - & - & - & - \\
\hline Ymin & -63.34 & -60.34 & 65.91 & NS & $2(20 \%)$ & $2(20 \%)$ & $2(20 \%)$ & NS \\
\hline Ymax & -45.29 & -40.39 & -50.23 & NS & $2(20 \%)$ & $2(20 \%)$ & $2(20 \%)$ & NS \\
\hline Ymed & -54.82 & -53.78 & -49.36 & NS & $2(20 \%)$ & $2(20 \%)$ & $3(30 \%)$ & - \\
\hline$S D(Y)$ & 0.40 & 0.41 & 0.40 & NS & - & - & - & NS \\
\hline L & 503.26 & 482.29 & 492.91 & NS & $2(20 \%)$ & $2(20 \%)$ & $2(20 \%)$ & NS \\
\hline S & 327.12 & 303.92 & 336.43 & NS & $3(30 \%)$ & $3(30 \%)$ & $3(30 \%)$ & NS \\
\hline FFTx & 0.08 & 0.08 & 0.08 & NS & - & - & - & - \\
\hline FFTy & 0.08 & 0.08 & 0.06 & NS & - & - & - & - \\
\hline VEL & 16.23 & 14.38 & 16.36 & NS & - & - & - & - \\
\hline SD(VEL) & 11.36 & 10.38 & 12.65 & NS & $5(50 \%)$ & $4(40 \%)$ & $4(40 \%)$ & NS \\
\hline
\end{tabular}




\begin{tabular}{|c|c|c|c|c|c|c|c|c|}
\hline \multicolumn{9}{|c|}{ CYSTS (N = 11) } \\
\hline & Pre-CE & Post-CE1 & Post-CE2 & p1 & Pre-CE N $(\%)$ & Post-CE1 N $(\%)$ & Post-CE2 $N^{\circ}(\%)$ & p2 \\
\hline Xmin & -4.28 & -2.89 & -4.35 & NS & 2 (18\%) & $1(9 \%)$ & 2 (18\%) & NS \\
\hline Xmax & 14.3 & 11.29 & 9.13 & NS & $2(18 \%)$ & $1(9 \%)$ & $1(9 \%)$ & NS \\
\hline Xmed & 6.16 & 5.29 & 3.69 & NS & $1(9 \%)$ & $1(9 \%)$ & $1(9 \%)$ & NS \\
\hline $\operatorname{SD}(X)$ & 0.25 & 0.26 & 0.28 & NS & - & - & - & - \\
\hline Ymin & -72.38 & -70.31 & -65.5 & NS & 0 & 0 & 0 & NS \\
\hline Ymax & -50.39 & -48.34 & -49.1 & NS & $2(18 \%)$ & $2(18 \%)$ & $2(18 \%)$ & NS \\
\hline Ymed & -62.14 & -59.29 & -57.49 & NS & $1(9 \%)$ & $1(9 \%)$ & $1(9 \%)$ & - \\
\hline$S D(Y)$ & 0.42 & 0.40 & 0.36 & NS & - & - & - & NS \\
\hline $\mathrm{L}$ & 501.34 & 490.29 & 505.39 & NS & $1(9 \%)$ & $1(9 \%)$ & $1(9 \%)$ & NS \\
\hline S & 298.56 & 257.82 & 282.31 & NS & $2(18 \%)$ & $1(9 \%)$ & $2(18 \%)$ & NS \\
\hline FFTx & 0.04 & 0.04 & 0.04 & NS & - & - & - & - \\
\hline FFTy & 0.04 & 0.04 & 0.03 & NS & - & - & - & - \\
\hline VEL & 12.76 & 12.08 & 12.23 & NS & - & - & - & - \\
\hline SD(VEL) & 10.89 & 11,31 & 11.41 & NS & $2(18 \%)$ & $2(18 \%)$ & $2(18 \%)$ & NS \\
\hline \multicolumn{9}{|c|}{ REINKE'S OEDEMA $(\mathrm{N}=4)$} \\
\hline & Pre-CE & Post-CE1 & Post-CE2 & $\mathrm{p} 1$ & Pre-CE $N^{\circ}(\%)$ & Post-CE1 Nº (\%) & Post-CE2 Nº (\%) & p2 \\
\hline Xmin & -11.91 & -10.93 & -10.6 & NS & 0 & 0 & 0 & NS \\
\hline Xmax & 19.18 & 18.92 & 15.19 & NS & 0 & 0 & 0 & NS \\
\hline Xmed & -0.48 & 1.39 & 1.21 & NS & $1(25 \%)$ & $1(25 \%)$ & $1(25 \%)$ & NS \\
\hline $\operatorname{SD}(X)$ & 0.45 & 0.41 & 0.36 & NS & - & - & - & - \\
\hline Ymin & -77.74 & -74.92 & -70.76 & NS & $1(25 \%)$ & $1(25 \%)$ & $1(25 \%)$ & NS \\
\hline Ymax & -26.40 & -25.93 & -32.98 & NS & 0 & 0 & $1(25 \%)$ & NS \\
\hline Ymed & -42.98 & -40.38 & -49.39 & NS & 0 & 0 & 0 & - \\
\hline$S D(Y)$ & 0.70 & 0.66 & 0.54 & NS & - & - & - & NS \\
\hline $\mathrm{L}$ & 612.27 & 603.38 & 598.49 & NS & $1(25 \%)$ & $1(25 \%)$ & $1(25 \%)$ & NS \\
\hline S & 321.78 & 355.98 & 343.05 & NS & 0 & 0 & 0 & NS \\
\hline FFTx & 0.12 & 0.12 & 0.14 & NS & - & - & - & - \\
\hline FFTy & 0.12 & 0.12 & 0.12 & NS & - & - & - & - \\
\hline VEL & 23.27 & 22.79 & 19.49 & NS & - & - & - & - \\
\hline SD(VEL) & 11.41 & 10.96 & 10.62 & NS & $2(50 \%)$ & $2(50 \%)$ & $2(50 \%)$ & NS \\
\hline
\end{tabular}

in posture. In fact, static stabilometry performed in basal conditions has been able to reveal postural modifications associated with voice disorders, which showed significant variations in the stabilometric parameters after speech rehabilitation ${ }^{313}$. On the other hand, there are no studies aimed at discovering whether these postural alterations are present even in cases of organic dysphonia and if removal of the lesion through phonomicrosurgery can, on its own, bring about substantial changes in postural performance. Hence, we submitted a heterogeneous group of patients with organic dysphonia (vocal fold polyps, nodules, acquired submucosal cysts and Reinke's oedema) to static stabilometry to see if there were any postural characteristics that were typical to the individual organic disorder, and to assess if phonomicrosurgery alone is sufficient to modify the posture of patients.

In basal conditions, none of the subgroups studied (pol- yps, cysts and Reinke's oedema) showed any characteristic pathological stabilometric pattern. In fact, the majority of parameters were within the normal range, with no significant deviations from the centre of gravity. Only eight patients $(8 / 10 ; 80 \%)$ in the subgroup with bilateral nodules on the vocal folds showed a forward shift from the centre of gravity, and pathological S and SD VEL values both in open-eye and closed-eye conditions, attesting the postural characteristics of subjects with pure dysfunctional dysphonia ${ }^{13}$. This confirms what has already been acknowledged; that is, vocal fold nodules may be, at least in some cases, the direct consequence of long-standing dysfunctional dysphonia that has been inadequately treated. Hence, patients with vocal fold nodules display a stabilometric pattern both in the open-eye and closed-eye examinations that is similar to what is seen in patients with pure dysfunctional dysphonia; on the contrary, however, the 
parameters of the former subjects do not improve significantly at 24 hours after surgery. While the majority of the stabilometric parameters improve significantly in subjects with pure dysfunctional dysphonia after rehabilitation therapy ${ }^{313}$, the group with vocal fold nodules continue to have modified posture at 24 hours and 6 months after phonosurgery and manifest no significant variations. From a postural point of view, this fact indirectly confirms the importance of giving adequate and sufficiently lengthy rehabilitation treatment to patients with dysfunctional dysphonia associated with altered posture. On the other hand, the static stabilometric results obtained both in basal conditions and after phonomicrosurgery in cases of polyps, cysts and Reinke's oedema demonstrate how modified postural performance in these circumstances is much less pronounced and not so distinctive. One would assume
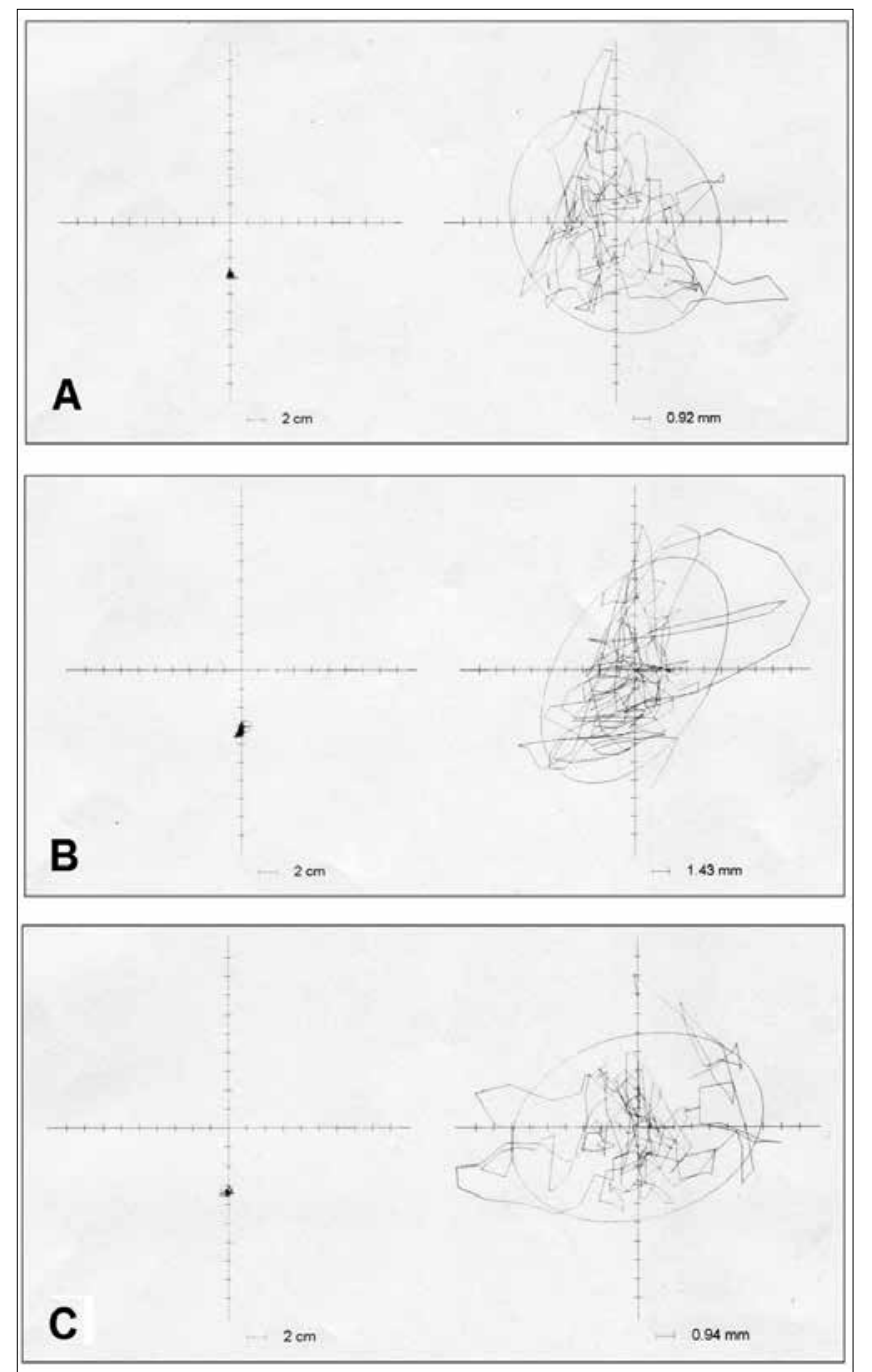

Fig. 1. Stabilometric finding (statokinesigram) of a patient with submucosal retention cyst in the closed-eye mode in basal conditions (A), $24 \mathrm{~h}$ after phonomicrosurgery (B) and 6 months after surgery (C). No characteristic pathological pattern was seen in basal conditions; a comparison performed before and after surgery (after 24h and after 6 months), revealed no significant difference in the statokinesigram pattern. that, immediately after surgery, the situation remains the same as before, while a longer follow-up can show some changes in postural control, related to voice amelioration. The follow-up at 6 months contradicts this hypothesis: in fact, the stabilometric parameters 6 months after surgery do not change in a statistically significant way as formerly observed at 24 hours after surgery (Fig. 1).

\section{Conclusions}

Thanks to static stabilometry it is possible to define the absence of postural alterations in subjects with organic disorders on the glottis plane that are not secondary to problems of a dysfunctional nature. Moreover, this study demonstrates how simple surgical removal of the vocal fold lesion does lead to modification of posture.

In spite of the fact that the results obtained refer to an extremely heterogeneous group, from both a pathophysiological and a clinical point of view, and that the statistical analysis was performed on a restricted number of subjects within each individual subgroup, it is important to again stress how important it is to submit dysphonic subjects with no signs of vertigo to stabilometric analysis.

This study is in line with what has been reported in previous studies ${ }^{313}$, namely, that skilfully performed speech rehabilitation therapy is fundamental for correcting both voice disorders and altered posture in cases of dysfunctional dysphonia. The results obtained in this study will have to be confirmed in the future, taking into account a larger patient population and a lengthier follow-up.

\section{References}

1 Koufman JA, Radomski TA, Joharii GM, et al. Laryngeal biomechanics of the singing voice. Otolaryngol Head Neck Surg 1996;115:527-37.

2 Kooijman PG, de Jong FI, Oudes MJ, et al. Muscular tension and body posture in relation to voice handicap and voice quality in teachers with persistent voice complaints. Folia Phoniatr Logop 2005;57:134-47.

3 Bruno E, De Padova A, Napolitano B, et al. Voice disorders and posturography: variables to define the success of rehabilitative treatment. J Voice 2009;23:71-5.

4 McKinney JC. Diagnosis and correction of vocal faults: a manual for teachers of singing and for choir directors. Revised edition. Nashville, TN: Genevox Music Group; 1994.

5 Brown OL. Discover your voice: how to develop healthy voice habits. San Diego, CA: Singular; 1996.

6 Schneider CM, Dennehy CA, Saxon KG. Exercise physiology principles applied to voice performance: the improvement of postural alignment. J Voice 1997;11:332-7.

7 Peckham A. The contemporary singer: elements of vocal technique. Boston, MA: Berklee Press; 2000.

8 Rubin JS, Mathieson L, Blake A. Posture and voice. J Singing 2004;60:271-5. 
9 Behrman A. Common practices of voice therapists in the evaluation of patients. J Voice 2005;19:454-69.

10 Herman-Ackah YD. Physiology of voice production: considerations for the vocal performer. J Singing 2005;62:173-6.

11 Grini MN, Ouaknine M, Giovanni A. Contemporary postural and segmental modification of forced voice. Rev Laryngol Otol Rhinol 1998;119:253-7.

12 Giovanni A, Assaiante C, Galmiche A, et al. [Vocal forcing and posture: experimental studies on normal subject]. Rev Laryngol Otol Rhinol (Bord) 2006;127:285-91.

13 Nacci A, Fattori B, Mancini V, et al. Posturographic analysis in patients with dysfunctional dysphonia before and after speech therapy/rehabilitation treatment. Acta Otorhinolaryngol Ital 2012;32:115-21.

14 Giovanni A, Akl L, Ouaknine M. Postural dynamics and vo- cal effort: preliminary experimental analysis. Folia Phoniatr Logop 2008;60:80-5.

15 Jacobson BH, Johnson A, Grywalski C, et al. The Voice Handicap Index (VHI): development and validation. Am J Speech Lang Pathol 1997;6:66-70.

16 Dejonckere PH, Remacle M, Fresnel-Elbaz E, et al. Differentiated perceptual evaluation of pathological voice quality: reliability and correlations with acoustic measurements. Rev Laryngol Otol Rhinol 1996;117:219-24.

17 Casolino D, Ricci Maccarini A. Fonochirurgia endolaringea. Quaderni monografici di aggiornamento AOOI. Pisa: Pacini Editore; 1997.

18 Dailey S. Diagnostic and Therapeutic pitfalls in phonosurgery. Otolaryngol Clin North Am 2006;39:11-22.

19 Huche F. La voix: pathologie vocale. Paris: Masson; 1990.

Received: January 13, 2016 - Accepted: November 18, 2016

Address for correspondence: Andrea Nacci, ENT Audiology Phoniatrics Unit, Department of Neurosciences, University of Pisa, via Paradisa 2, 56124 Pisa, Italy. Tel. +39 050 997518. Fax +39050 997 548. E-mail: a.nacci@med.unipi.it 